\title{
Scalar Fluctuations from Extended Non-equilibrium Thermodynamic States
}

\author{
R. E. Nettleton \\ Department of Physics, University of the Witwatersrand, Johannesburg, South Africa
}

Z. Naturforsch. 40 a, 976-985 (1985); received July 30, 1985

\begin{abstract}
In the framework of extended non-equilibrium thermodynamics, the local non-equilibrium state of a liquid is described by the density, temperature, and a structural variable, $\zeta$, and its rateof-change. $\zeta$ is the ensemble average of a function $A(Q)$ of the configuration co-ordinates, and it is assumed to relax to local equilibrium in a time short compared to the time for diffusion of an appreciable number of particles into the system. By a projection operator technique of Grabert, an equation is derived from the Liouville equation for the distribution of fluctuations in $N$, the particle number, and in $A$ and $\dot{A}$. An approximate solution is proposed which exhibits nonequilibrium corrections to the Einstein function in the form of a sum of thermodynamic forces. For a particular structural model, the corresponding non-Einstein contributions to correlation functions are estimated to be very small. For variables of the type considered here, the thermodynamic pressure is found to equal the pressure trace.
\end{abstract}

\section{Introduction}

In order to develop a mesoscopic theory applicable to states far from equilibrium or, as in the present development, a time-scale shorter than hydrodynamic, an extended non-equilibrium thermodynamics has been formulated [1-4] which postulates that a local entropy, $S$, or free energy, $F$, may be defined for a small subvolume selected for study in a continuous fluid so that it depends on the dissipative fluxes as state variables, as well as on mass density, $\varrho$, and temperature, $T$. These ideas are similar to earlier work [5-9] which explored the congruence of a theory based on these assumptions with the Onsager-Casimir formalism and showed that the extended formalism led to an extended set of reciprocity relations useful for calculating liquid transport coefficients. Since the mesoscopic state variables are averages over a time-dependent ensemble, there will be fluctuations in this ensemble from these mean values. For want of a more general approach, the Einstein function $C \exp \delta^{2} S / \varkappa$ has been used $[10,11]$ to give the probability distribution of these fluctuations, where $x$ is Boltzmann's constant, $\delta^{2} S$ the second-order variation in the extended entropy, and $C$ the normalization. In the present paper we wish to generalize these calcula-

Reprint requests to Dr. R. E. Nettleton, Department of Physics, University of the Witwatersrand, I Jan Smuts Avenue, Johannesburg 2001, South Africa. tions in two ways. Firstly, scalar variables suggested by earlier work on sound absorption in simple liquids, representing a structural parameter and its time-derivative, will be used rather then the vector heat flux and internal energy. Secondly, a FokkerPlanck equation derived by Grabert [12] will be used to calculate the fluctuation distribution, yielding a result more general than the Einstein function and providing a systematic way of correcting the latter. The coupling of fluctuations in the mass density will also be included. Although the phenomenology is extended to short times, we do not consider highly non-linear phenomenological equations for which the approximations used would be invalid.

In utilising the formalism of Grabert, we are illustrating for the case of scalar variables a calculation previously [17] worked out for the case where the fast variable was the vector heat flux and the slow variable was the temperature. The role played by $\nabla T$, which characterises the coupling to the surroundings in that formalism, is analogous to that played by $\nabla \cdot \boldsymbol{u}$ in the present case, where $\boldsymbol{u}$ is the mass velocity. We find that the Einstein distribution is not exact even when $\nabla \cdot \boldsymbol{u}=0$.

To correct the Einstein distribution, we derive a Fokker-Planck-type equation for the fluctuation distribution, using a projection operator technique originally developed by Zwanzig [13,14] and extended by Grabert [12]. In this approach, we have a number of state variables $\alpha_{j}=\left\langle A_{j}(\boldsymbol{Q}, \boldsymbol{P})\right\rangle$ which are 
ensemble averages of dynamical functions $A_{j}$ of the configuration co-ordinates $\boldsymbol{Q}$ and momenta $\boldsymbol{P}$ of the particles in the system. A distribution function,

$$
g\left(\left\{\alpha_{j}\right\}\right)=\int f_{N} \prod_{j} \delta\left(\alpha_{j}-A_{j}\right) \mathrm{d} \Gamma
$$

is then obtained for the probability distribution of the $\alpha_{j}$, from the solution, $f_{N}$, of the Liouville equation. A Fokker-Planck equation for $g$ is derived [14] by operating with the projection operator, proportional to the product of Dirac deltas in (1), on the Liouville equation itself. In the extended non-equilibrium thermodynamics, the set $\left\{\boldsymbol{\alpha}_{j}\right\}$ is augmented by an additional set of independent state variables $\left\{\eta_{j}\right\}=\left\{\dot{\alpha}_{j}\right\}=\left\{\left\langle i L A_{j}\right\rangle\right\}$, where $L$ is the self-adjoint Liouville operator. To accomodate this extension, the Fokker-Planck equation obtained by Zwanzig [14] was generalized [15] to yield an equation for the joint distribution, $g\left(a_{j}, v_{j}\right)$ for the probability that the $A_{j}$ have values between $a_{j}$ and $a_{j}+\mathrm{d} a_{j}$ while the $\dot{A}_{j} \equiv i L A_{j}$ have values between $v_{j}$ and $v_{j}+\mathrm{d} v_{j}$. The first moments of this generalized Fokker-Planck equation were shown [15] to yield the phenomenological equations of extended irreversible thermodynamics.

The previous derivations $[14,15]$ of equations for $g$ imagined a system isolated from its surroundings, in which the approach to equilibrium was governed entirely by irreversible processes within the system. If we wish to describe a small sub-volume immersed in an infinite fluid, the system is not closed, and the equation for $g$ must be augmented by terms arising from interaction with the surroundings, which may be regarded as reservoirs of heat and particles. These terms depend on the state of the surroundings relative to that of the system and therefore on the gradients of temperature [16] and fluid velocity. Furthermore, the equilibrium distribution is not microcanonical, as in the earlier treatments $[14,15]$, but rather grand canonical. To provide for exchange of heat with the surroundings, Grabert [12] has defined a more general projection operator leading to a $g$ corresponding in equilibrium to the canonical ensemble. In the present paper, we generalize this formalism still further to provide for exchange of particles with the surroundings and specialize it to the case where there is a single structural variable, $\zeta$, and the local state is described by the set $\varrho, T, \zeta$, $\eta \equiv \dot{\zeta}$. The resulting Fokker-Planck equation can be solved for the distribution $g$ in which fluctuations in the values of the dynamical functions $A$ and $\dot{A}$ are coupled to fluctuations in the density.

In the following section we shall give a more precise characterization of the model and then go on in Sect. 2.2 to derive the equation for the distribution, $g$. In Sect. 3.1, we propose an ansatz for the solution, which reduces to the Einstein function in equilibrium. The ansatz is a sum of thermodynamic forces with coefficients which are sums of Hermite polynomials, chosen to satisfy the normalization and the consistency condition that the first moment of $g$ are the ensemble averages, $\zeta$ and $\eta$. In Sect. 3.2, we substitute the ansatz into the FokkerPlanck equation and derive equations for the constant coefficients. In Sect. 3.3 we calculate correlation functions, and the solution is specialized to times long compared with the relaxation times for $\zeta$ and $\eta$, where the corrections to the Einstein function are proportional to $\nabla \cdot \boldsymbol{u}$, the divergence of the fluid velocity $\boldsymbol{u}$. Numerical estimates of these corrections are made in Sect. 4 for a model in which $\zeta$ is the volume fraction of "holes", or local regions where the intermolecular separation is sufficiently great to permit self-diffusion. These estimates indicate that the corrections to the Einstein distribution are, for this model, negligibly small, although the model and approximations are crude, making the estimates rough. In conjunction with Sect. 4, a method is required for calculating the extended entropy, and this is described in the Appendix. The similarities between the present results and corresponding calculations $[16,17]$ for vector variables will be discussed in Section 5. There we shall show that, for a structural variable $\zeta$, the kinetic equation has no term proportional to $\nabla \cdot \boldsymbol{u}$. This implies equality, in this case, of the thermodynamic and hydrodynamic pressure.

\section{Derivation of the Fokker-Planck Equation}

The system to be considered is a small cube, of side $l$, immersed in an infinite, continuous liquid phase which may be replaced by reservoirs of heat and particles. The magnitude of $l$ must be small in comparison with the wavelengths of macroscopic disturbances such as sound waves which propagate in the surrounding medium. If $\boldsymbol{u}(\boldsymbol{x})$ is the fluid velocity in the centre of the system, the reservoirs at $\boldsymbol{x} \pm \frac{1}{2} \boldsymbol{l} \hat{\boldsymbol{r}}$, where $\hat{\boldsymbol{r}}$ is a unit vector along one of the 
Cartesian axes, will supply particles with a velocity $\boldsymbol{u}\left(\boldsymbol{x} \pm \frac{1}{2} l \hat{\boldsymbol{r}}\right)$. We shall suppose that the thermodynamic state variables comprise, in addition to $\varrho$ and $T$, a single structural variable $\zeta=\langle A(Q)\rangle$ plus its rate-of-change, $\eta=\dot{\zeta}=\langle i L A\rangle$, where the angular brackets are averages in the time-dependent ensemble. In Sect. 2.1, we give an example of such a variable, based on a model to be used in the numerical estimates of Section 4. The important properties of $A(Q)$ are that it depends only on $Q$ and has a short relaxation time. For times short compared with this time, the system is described by a number of mesoscopic local state variables, such as $\eta$ and $\zeta$, which relax to functions of $\varrho, T$, and derivatives of $\boldsymbol{u}$. Over longer time scales, the macroscopic variables $\varrho, T$, and $\boldsymbol{u}$ evolve according to the laws of hydrodynamics. For this model, we proceed in Sect. 2.2 to derive the equation for $g$.

\subsection{Characterization of the Model}

A structural model having the characteristics required for the present treatment is found in the hole theory of liquids, used [18] to calculate the structural contribution to sound absorption in a nonassociated liquid. In this model, a molecule may find itself in a region where the mean intermolecular separation is sufficient to permit self-diffusion and consequent structural rearrangement. We can thus take $A(Q)$ to be the fraction of molecules whose mean distances to their neighbours exceed the critical value for self-diffusion.

To estimate the relaxation time for this model, we observe that molecular motion in a liquid, over a time sufficiently short that a molecule stays imprisoned in a cage formed by its neighbours, can be viewed as a superposition of hypersound waves. The expansion of a region of diameter $\delta$, the mean intermolecular separation, results from waves of wavelength $\sim \delta$ which have a mean frequency $\bar{v}_{1}$. A calculation has been made [19] of the probability $Q_{1}$ that the wave amplitudes add to give a value $\delta \varepsilon$ necessary to permit a molecule to diffuse out of its cage. The probability per unit time that this will occur is $\bar{v}_{1} Q_{1}$.

By requiring balance in equilibrium of the processes of creation and destruction of "holes", one finds [18] that the relaxation time for $\zeta$ obeys $\tau_{1}^{-1}=\bar{v}_{1} Q_{1} /\left[\zeta_{0}\left(1-\zeta_{0}\right)\right]$ where $\zeta_{0}$ is the equilibrium concentration of holes. An estimate of $\zeta_{0}$ has been made [18] by treating $\delta$ as an oscillator and calculating the probability that the displacement of the latter exceeds $\delta \varepsilon$. This gives the fraction of the time a given region is critically-expanded which is identified, in equilibrium, with the volume fraction $\zeta_{0}$ of holes. Details of the application of these results to $\mathrm{Ar}$ are given in the Appendix. The dependence of the shear modulus on $\zeta$ is estimated from the expression appropriate to a solid with gas-like spherical inclusions, as suggested in the original development [19] of this model. This yields results which are reasonable as to sign and order-of-magnitude (see Section 4). An estimate $\tau_{1} \sim 10^{-13} \mathrm{~s}$ has been made [18], and so $\zeta$ and $\eta$ for this kind of model may be regarded as fast variables which relax toward local equilibrium in a time short compared with the time required for exchange of appreciable numbers of particles with the surroundings.

Guided by the foregoing example, we shall suppose that $A(\boldsymbol{Q})$ is so defined that it is indeed a fast variable, with the consequence that the contributions to the equation for $g$ arising from relaxation processes within the system are the same as those we can calculate from the formalism of Grabert [12] using the Liouville equation for a fixed number $N$ of particles. To take into account the effect on the fast variables of fluctuations in density, we then augment the equation derived for fixed $N$ by adding a term proportional to $\nabla \cdot \boldsymbol{u}$, so devised that its first two moments are phenomenologically correct. This procedure is similar to that employed [16] to take into account fluctuations in heat flow when a temperature gradient is present. Referring to that treatment, we can see that if we calculate the contribution to the equation for $g$ from the reservoir at $\boldsymbol{x}+\frac{1}{2} l \hat{\boldsymbol{r}}$, this will be a function of $\boldsymbol{u}\left(\boldsymbol{x}+\frac{1}{2} l \hat{\boldsymbol{r}}\right)$ while that from the reservoir at $\boldsymbol{x}-\frac{1}{2} l \hat{\boldsymbol{r}}$ will be a function of $\boldsymbol{u}\left(\boldsymbol{x}-\frac{1}{2} l \boldsymbol{r}\right)$. The net contribution from the two reservoirs should depend on $\nabla \cdot \boldsymbol{u}$. We shall neglect temperature and heat flow fluctuations here and assume $\nabla T=0$.

\subsection{Derivation of the Fokker-Planck Equation}

Since the system can exchange both energy and particles with its surroundings, it is described microscopically by a time-dependent grand ensemble. The joint probability amplitude for finding the system with size $N$ and at point $\Gamma$ in the $N$ - 
particle phase space is

$$
f(N, \Gamma)=f_{1}(N) f(\Gamma \mid N) .
$$

The distribution $f(\Gamma \mid N)$ satisfies the $N$-particle Liouville equation. Since $\zeta$ is a fast variable which relaxes to local equilibrium in a time short compared with the time required for an appreciable change in $N(\nabla \cdot \boldsymbol{u}$ assumed small $)$, this relaxation will be governed by $f(\Gamma \mid N)$.

Associated with the microscopic distribution $f(\Gamma \mid N)$ we have, as a special case of (1),

$$
g\left(v, v_{1} \mid N\right)=\int f(\Gamma \mid N) \delta(A-v) \delta\left(\dot{A}-v_{1}\right) \mathrm{d} \Gamma,
$$

so defined that $g\left(v, v_{1} \mid N\right) \mathrm{d} v \mathrm{~d} v_{1}$ is the probability that $A$ has a value within $\mathrm{d} v$ of $v$ and $\dot{A} \equiv i L A$ within $\mathrm{d} v_{1}$ of $v_{1}$. An equation for $g$ has been derived [15] by a projection operator technique for the case of a closed system microcanonically-distributed in equilibrium. This has been generalized by Grabert [[12]. Eq. (4.4.7)], to assume the form:

$$
\begin{aligned}
\frac{\partial}{\partial t} g\left(r, v_{1} N\right)= & -\frac{\partial}{\partial r}\left(q_{A} g\right)-\frac{\partial}{\partial \iota_{1}}\left(q_{A 1} g\right) \\
& +\frac{\partial}{\partial v_{1}}\left[D p_{\beta}\left(v, v_{1}\right) \frac{\partial}{\partial v_{1}}\left(g / p_{\beta}\right)\right],
\end{aligned}
$$

where

$$
\begin{aligned}
q_{A} \equiv & \left(Z p_{\beta}\right)^{-1} \int \delta(A-v) \delta\left(\dot{A}-v_{1}\right) \\
& \cdot \dot{A} e^{-\beta H} \mathrm{~d} \Gamma=v_{1} \\
q_{A 1} \equiv & \left(Z p_{\beta}\right)^{-1} \int \delta(A-v) \delta\left(\dot{A}-v_{1}\right) \\
& \cdot A^{+} e^{-\beta H} \mathrm{~d} \Gamma \\
A^{+} \equiv & (i L)^{2} A \\
D p_{\beta} \equiv & \int_{0}^{\infty} \mathrm{d} s \int \mathrm{d} \Gamma\left\{\left[A^{+}(s)-q_{A 1}\right]\left[A^{+}-q_{A 1}\right] e^{-\beta H}\right. \\
& \cdot \delta(A-v) \delta\left(\dot{A}-v_{1}\right)
\end{aligned}
$$

This formalism provides for the fact that, for an open system in equilibrium, $f(\Gamma \mid N)=\varrho_{\beta}=$ $Z^{-1} e^{-\beta H}$, where $Z$ is the $N$-particle canonical partition function. $p_{\beta}$ is the limit approached by $g$ as $f(\Gamma \mid N) \rightarrow \varrho_{\beta}$.

The first moments of (4a) give the phenomenological equations for $\zeta$ and $\eta$ in the form (cf. [15])

$$
\begin{aligned}
& \zeta=\int g\left(v, v_{1}\right) v \mathrm{~d} v \mathrm{~d} v_{1}, \\
& \eta=\int g\left(v, v_{1}\right) v_{1} \mathrm{~d} v \mathrm{~d} v_{1}, \\
& \dot{\zeta}=\eta, \\
& \dot{\eta}=L_{\zeta} F_{\zeta}-2 \mu_{\eta} L_{\eta} \eta,
\end{aligned}
$$

where $F_{\zeta}=-2 \mu_{\zeta}\left(\zeta-\zeta_{0}\right)=\partial S / \partial \zeta$ and $-2 \mu_{\eta} \eta=$ $\partial S / \partial \eta$ are thermodynamic forces conjugate to the fluxes $\dot{\zeta}$ and $\dot{\eta}$, respectively, and $S$ [see (7) below] is the entropy for the non-equilibrium state defined by the variables $\varrho, T, \zeta, \eta$ (cf. [15], Eqs. (15) and (16)). The phenomenological coefficient $L_{\eta}=D / x$ ([15], Eqs. (16), (33)), while $L_{\zeta}$ and $L_{\eta}$ are related by the Casimir anti-reciprocity relation [15].

$$
L_{\zeta}=1 / 2 \mu_{\eta} .
$$

To effect a further reduction of $(4 \mathrm{a})$ consistent with the approximations, e.g. neglect of $0\left(\dot{A}^{3}\right)$, which lead to the phenomenological equations $(5 \mathrm{c}, \mathrm{d})$ of extended irreversible thermodynamics, we need to evaluate $q_{A 1}$. By effecting a partial integration in $(4 \mathrm{c})$, equivalent to moving one of the operators $i L$ in $A^{+}$so that it operates on the Dirac deltas (cf. [15], Eq. (23)), we obtain to $O\left(\dot{A}^{3}\right)$

$$
\begin{aligned}
q_{A 1} & =v_{1}^{2} \frac{\partial}{\partial v} \ln \int \varrho_{\beta} \delta(A-v) \delta\left(\dot{A}-v_{1}\right) \mathrm{d} \Gamma \\
& =\varkappa^{-1} v_{1}^{2} \partial S / \partial v=-\varkappa^{-1} v_{1}^{2} 2 \mu_{\zeta}\left[v-\zeta_{0}(N, T)\right],
\end{aligned}
$$

where $S$ is the entropy given $A=v, \dot{A}=v_{1}$. Similar arguments show that, with neglect of $O\left(\dot{A}^{3}\right)$, the diffusion term in $(4 \mathrm{a})$ assumes the form

$$
D p_{\beta}\left(\partial^{2} / \partial v_{1}^{2}\right)\left(g / p_{\beta}\right) \text {. }
$$

To include density fluctuations, which are slow, alongside the fast fluctuations in $A$ and $\dot{A}$, we must determine the function,

$$
\begin{aligned}
g\left(N, v, v_{1}\right) & =\int f(N, \Gamma) \delta(A-v) \delta\left(\dot{A}-v_{1}\right) \mathrm{d} \Gamma \\
& =f_{1}(N) g\left(v, v_{1} \mid N\right) .
\end{aligned}
$$

The equation for $g\left(N, v, v_{1}\right)$ should reduce to that for $g\left(v, v_{1} \mid N\right)$ when diffusion across the boundaries can be neglected, and so we expect that $g\left(N, v, v_{1}\right)$ obeys the Fokker-Planck equation augmented by a term proportional to the rate of diffusion. The structure of this term is determined phenomenologically in part by the requirement that $g$ be normalized, so that the zeroth moment of the additional term vanishes. In addition $\int g N \mathrm{~d} N \mathrm{~d} v \mathrm{~d} v_{1}$ $=N_{0}$, the ensemble average, which obeys the continuity equation. The required properties are found in the equation

$$
\begin{aligned}
\frac{\partial g}{\partial t}= & -\frac{\partial}{\partial v}\left[v_{1} g\right]-\frac{\partial}{\partial v_{1}}\left[q_{A 1} g\right] \\
& +D p_{\beta} \frac{\partial^{2}}{\partial v_{1}^{2}}\left(g / p_{\beta}\right)+\frac{\varrho l^{3}}{m} \frac{\partial g}{\partial N} \nabla \cdot \boldsymbol{u},
\end{aligned}
$$

where $\varrho=N_{0} l^{-3} m, m$ being the particle mass. 


\section{Calculation of the Distribution}

\subsection{Ansatz for $g$}

In equilibrium the $v$ - and $v_{1}$-dependence of $g$ should be given in lowest approximation by the Einstein distribution, $\exp \left(\partial^{2} S / x\right)$ for a system of fixed size $N$. This corresponds to

$$
\begin{aligned}
p_{\beta}= & C \exp \left\{-\mu_{\zeta} \varkappa^{-1}\left(v-\zeta_{0}(N)\right)^{2}-\mu_{\eta} \varkappa^{-1} v_{1}^{2}\right\} \\
& \cdot\left[1+\mu_{v \zeta}\left(v-\zeta_{0}(N)\right) v_{1}^{2}+\ldots\right],
\end{aligned}
$$

where the ellipsis refers to higher-order terms in $v-\zeta_{0}$ and $v_{1}$ which do not contribute explicitly to the calculations described below. The coefficients $\tilde{\mu}_{o \eta}$ and $\tilde{\mu}_{\zeta \eta}$ in (19) should be proportional to $\mu_{r \zeta}$. We have

$$
\begin{aligned}
v-\zeta_{0}(N)= & (v-\zeta)+\left(\zeta-\zeta_{0}\left(N_{0}\right)\right) \\
& -\zeta_{0 N} \cdot\left(N-N_{0}\right),
\end{aligned}
$$

where $\zeta_{0 N} \equiv \partial \zeta_{0}\left(N_{0}\right) / \partial N_{0}$. Substitution from (11) into (10), coupled with the hypothesis that the $v$ and $v_{1}$-dependence of the leading term in $g$ reduces to that of $p_{\beta}$ in equilibrium, leads to the conclusion that there will be correlations between fluctuations in $N$ and $A$.

When density fluctuations are included, the factor $p_{\beta}$ is multiplied by the equilibrium distribution for fluctuations in $N$ which is simply the grand canonical distribution, peaked about the ensemble average, $N_{0}$. Thus if $g$ is assumed to be the product of a Gaussian factor, $g^{(0)}$, multiplying a sum which includes higher-order corrections, we can postulate that

$$
\begin{aligned}
g^{(0)}= & C \exp \left\{-\left(\mu_{0} / \varkappa\right)\left(N-N_{0}\right)^{2}-\left(\mu_{\zeta} / \varkappa\right)(v-\zeta)^{2}\right. \\
& +\left(2 \mu_{\zeta} \zeta_{0 N} / \varkappa\right)(v-\zeta) \\
& \left.\cdot\left(N-N_{0}\right)-\left(\mu_{\eta} / \varkappa\right)\left(v_{1}-\eta\right)^{2}\right\},
\end{aligned}
$$

where $\mu_{Q}$ is a constant calculable in principle from the grand canonical $N$-distribution (see Appendix). No terms involving $\zeta-\zeta_{0}$ appear in $g^{(0)}$, since these are lumped with higher-order terms proportional to the thermodynamic force, $F_{\zeta}$, in the general ansatz for $g$.

To simplify the calculation, we introduce the reduced fluctuation variables

$$
\begin{aligned}
\xi & \equiv\left(\mu_{Q} / x\right)^{1 / 2}\left(N-N_{0}\right), \\
v & \equiv\left(\mu_{\zeta} / x\right)^{1 / 2}(v-\zeta), \\
z & \equiv\left(\mu_{\eta} / \varkappa\right)^{1 / 2}\left(v_{1}-\eta\right),
\end{aligned}
$$

Next, we can complete the square by defining

$$
\begin{aligned}
\sigma_{\xi v} & \equiv 2\left(\mu_{\zeta} / \mu_{\varrho}\right)^{1 / 2} \zeta_{0 N}, \\
\tau_{1} & \equiv 1+\frac{1}{2} \sigma_{\xi v}, \\
\tau_{2} & \equiv 1-\frac{1}{2} \sigma_{\xi v}, \\
x & \equiv\left(\tau_{2} / 2\right)^{1 / 2}(\xi+v), \\
y & \equiv\left(\tau_{1} / 2\right)^{1 / 2}(\xi-v) .
\end{aligned}
$$

In terms of these variables,

$$
g^{(0)}=\pi^{-3 / 2} \exp \left(-x^{2}-y^{2}-z^{2}\right) .
$$

This $g^{(0)}$ is the pre-factor of a more general ansatz

$$
g=g^{(0)}[1+G(x, y, z)],
$$

where $G$ must be constructed to satisfy the normalization condition

$$
\int_{-\infty}^{\infty} g \mathrm{~d} x \mathrm{~d} y \mathrm{~d} z=1
$$

and the consistency conditions

$$
\begin{aligned}
\int g x \mathrm{~d} x \mathrm{~d} y \mathrm{~d} z & =\int g y \mathrm{~d} x \mathrm{~d} y \mathrm{~d} z \\
& =\int g z \mathrm{~d} x \mathrm{~d} y \mathrm{~d} z=0 .
\end{aligned}
$$

These conditions are satisfied by an expansion in Hermite polynomials $H_{i}$ :

$$
\begin{aligned}
G= & \tilde{\mu}_{\varrho 3} H_{3}(x)+\tilde{\mu}_{\varrho 5} H_{5}(x)+\tilde{\mu}_{\zeta 3} H_{3}(y)+\tilde{\mu}_{\zeta 5} H_{5}(y) \\
& +H_{3}(x)\left[\tilde{\mu}_{\varrho v} y^{2}+\tilde{\mu}_{\varrho \eta} z^{2}\right]+H_{3}(y)\left[\tilde{\mu}_{\zeta \varrho} x^{2}+\tilde{\mu}_{\zeta \eta} z^{2}\right] \\
& +F_{\zeta}\left[A_{1} H_{2}(x)+A_{2} H_{2}(y)+A_{3} H_{2}(z)+D_{1} x y\right. \\
& \left.+D_{2} y z+D_{3} z x\right] \\
+ & \eta\left[B_{1} H_{2}(x)+B_{2} H_{2}(y)+B_{3} H_{2}(z)+E_{1} x y\right. \\
& \left.+E_{2} y z+E_{3} z x\right] \\
+ & \nabla \cdot \boldsymbol{u}\left[F_{1} H_{2}(x)+F_{2} H_{2}(y)+F_{3} H_{2}(z)\right. \\
& \quad+F_{4} H_{2}(x) z^{2}+F_{5} H_{2}(y) z^{2}+G_{1} x y \\
& \left.+G_{2} y z+G_{3} z x+G_{4} x y z^{2}\right]+\ldots
\end{aligned}
$$

\subsection{Determination of the Coefficients}

If the ansatz defined by (16) and (19) is substituted into (9), we want to show that the constant coefficients, $A_{1}, \ldots, G_{4}$, can be so determined that the Fokker-Planck equation is satisfied to a given degree of approximation. The terms in the ansatz go out to products of the forces, $F_{\zeta}, \eta$, and $\nabla \cdot \boldsymbol{u}$ with terms of second-order in $x, y, z$. The coefficients can be found by equating terms of the same type and order on each side of (9), after the ansatz has been substituted into the latter equation. 
By comparing coefficients of terms linear in $\eta, F_{\zeta}$, $\eta x^{2}, \eta y^{2}, F_{\zeta} x^{2}$, and $F_{\zeta} y^{2}$ on each side of (9) after substitution of (19), we find

$$
\begin{aligned}
B_{1}=0=B_{2}, & \\
A_{1}= & (3 / 2)\left(2 \varkappa \mu_{\zeta}\right)^{-1 / 2}\left[2 \tau_{2}^{1 / 2}\left(\tilde{\mu}_{\varrho 3}-20 \tilde{\mu}_{\varrho 5}\right)\right. \\
& \left.\quad+\tau_{1}^{1 / 2} \tilde{\mu}_{\zeta \varrho}\right], \\
A_{2}= & -(3 / 2)\left(\tau_{2} / 2 \varkappa \mu_{\zeta}\right)^{1 / 2} \tilde{\mu}_{\varrho v}, \\
A_{3}= & -B_{3}\left(8 L_{\eta} \mu_{\eta}^{2}\right)^{-1}-\varkappa \mu_{r \zeta}\left(8 \mu_{\eta} \mu_{\zeta}\right)^{-1}, \\
B_{3}= & {\left[4 L_{\eta} \mu_{\eta}+\mu_{\zeta} /\left(2 L_{\eta} \mu_{\eta}^{2}\right)\right]^{-1} } \\
\cdot & \left\{6 ( \mu _ { \zeta } / 2 \varkappa ) ^ { 1 / 2 } \left[2 \tau_{2}^{1 / 2}\left(\tilde{\mu}_{\varrho 3}-20 \tilde{\mu}_{\varrho}\right)\right.\right. \\
& \left.\left.+\tau_{1}^{1 / 2} \tilde{\mu}_{\zeta \varrho}-\tau_{2}^{1 / 2} \tilde{\mu}_{\varrho v}\right]-\left(\varkappa \mu_{r: \zeta} / 2 \mu_{\eta}\right)\right\} .
\end{aligned}
$$

In similar fashion, by comparing coefficients of terms in $\eta z^{2}$ and $F_{\zeta} z^{2}$ we get expressions for $D_{1}$ and $E_{1}$ while from terms in $F_{\zeta} y z$ and $F_{\zeta} z x$ we evaluate $E_{2}$ and $E_{3}$. Terms linear in $\eta y z$ and $\eta z x$ yield equations determining $D_{2}$ and $D_{3}$, while terms proportional to $\nabla \cdot \boldsymbol{u}$ and products of $\nabla \cdot \boldsymbol{u}$ with $x^{2}$, $x y$, and so on give equations relating $F_{i}$ and $G_{i}$ $(i=1, \ldots, 3)$ to the set $\left\{A_{i}\right\}$ and to $D_{2}$. These lengthy expressions will be omitted here. For a hole model, the probability of a fluctuation in the concentration of holes depends on the local density and, to a good approximation, should not be affected by a slow diffusion of particles into the system. In this approximation, we neglect the contribution of $\nabla \cdot \boldsymbol{u}$ to $\left\langle v^{2}\right\rangle$ and set (see below (23a))

$$
F_{1}=-\left(\tau_{2} / \tau_{1}\right) F_{2}+\frac{1}{4} G_{1}\left(\tau_{2} / \tau_{1}\right)^{1 / 2} .
$$

Fluctuations in $N$ will affect fluctuations in $\eta$. The latter, however, is a fast variable whose fluctuations are short compared to the time required for appreciable changes in $N$. Accordingly, it is reasonable to neglect the contribution of $\nabla \cdot \boldsymbol{u}$ to $\langle\xi z\rangle$ and set (cf. Eq. (23e))

$$
G_{2} \tau_{2}^{1 / 2}=-G_{3} \tau_{1}^{1 / 2} \text {. }
$$

\subsection{Correlation Functions}

To examine the structure of the correlations, we calculate them from the ansatz for $g$ :

$$
\begin{aligned}
& \left.\begin{array}{l}
\left\langle\xi^{2}\right\rangle \\
\left\langle v^{2}\right\rangle
\end{array}\right\}=\frac{1}{4}\left\{\mathrm{t}_{1}^{-1}+\tau_{2}^{-1}\right. \\
& +F_{\zeta}\left[4\left(A_{1} \tau_{2}^{-1}+A_{2} \tau_{1}^{-1)} \pm D_{1}\left(\tau_{1} \tau_{2}\right)^{-1 / 2}\right]\right. \\
& +\eta\left[4\left(B_{1} \tau_{2}^{-1}+B_{2} \tau_{1}^{-1}\right) \pm E_{1}\left(\tau_{1} \tau_{2}\right)^{-1 / 2}\right] \\
& \left.+\nabla \cdot \boldsymbol{u}\left[4\left(F_{1} \tau_{2}^{-1}+F_{2} \tau_{1}^{-1}\right) \pm G_{1}\left(\tau_{1} \tau_{2}\right)^{-1 / 2}\right]\right\} \text {, }
\end{aligned}
$$

$$
\begin{aligned}
&\langle\zeta v\rangle=\frac{1}{4}\left\{\tau_{1}^{-1}-\tau_{2}^{-1}+4\left[F_{\zeta}\left(A_{1} \tau_{2}^{-1}-A_{2} \tau_{1}^{-1}\right)\right.\right. \\
&+\eta\left(B_{1} \tau_{2}^{-1}-B_{2} \tau_{1}^{-1}\right) \\
&\left.\left.+\nabla \cdot \boldsymbol{u}\left(F_{1} \tau_{2}^{-1}-F_{2} \tau_{1}^{-1}\right)\right]\right\}, \\
&\left\langle z^{2}\right\rangle=\frac{1}{2}+2\left[A_{3} F_{\zeta}+B_{3} \eta+F_{3} \nabla \cdot \boldsymbol{u}\right], \\
&\langle\xi z\rangle=\left(2^{-1 / 2} / 4\right)\left[F_{\zeta}\left(D_{3} \tau_{2}^{-1 / 2}+D_{2} \tau_{1}^{-1 / 2}\right)\right. \\
&+\eta\left(E_{3} \tau_{2}^{-1 / 2}+E_{2} \tau_{1}^{-1 / 2}\right) \\
&\left.+\nabla \cdot \boldsymbol{u}\left(G_{3} \tau_{2}^{-1 / 2}+G_{2} \tau_{1}^{-1 / 2}\right)\right], \\
&\langle v z\rangle=\left(2^{-1 / 2} / 4\right)[ F_{\zeta}\left(D_{3} \tau_{2}^{-1 / 2}-D_{2} \tau_{1}^{-1 / 2}\right) \\
&+\eta\left(E_{3} \tau_{2}^{-1 / 2}-E_{2} \tau_{1}^{-1 / 2}\right) \\
&\left.+\nabla \cdot \boldsymbol{u}\left(G_{3} \tau_{2}^{-1 / 2}-G_{2} \tau_{1}^{-1 / 2}\right)\right] .
\end{aligned}
$$

In the present model, we cannot produce a genuinely steady state, since $\nabla \cdot \boldsymbol{u}$ cannot be held constant. However, $\nabla \cdot \boldsymbol{u}$ can remain approximately constant over a time much longer than the relaxation time for $A$. In this limit, from $(5 \mathrm{c}, \mathrm{d})$ :

$$
\begin{aligned}
\eta & \cong \dot{\zeta}_{0}=-\varrho\left(\partial \zeta_{0} / \partial \varrho\right) \nabla \cdot \boldsymbol{u}, \\
& F_{\zeta} \cong 2 \mu_{\eta}\left(L_{\eta} / L_{\zeta}\right) \eta \\
& \cong-2 \mu_{\eta}\left(L_{\eta} / L_{\zeta}\right) \varrho\left(\partial \zeta_{0} / \partial \varrho\right) \nabla \cdot \boldsymbol{u} .
\end{aligned}
$$

Substitution into $(23 \mathrm{a}-\mathrm{e})$ shows that the non-equilibrium corrections are all proportional to $\nabla \cdot \boldsymbol{u}$ in this quasi-steady state. These expressions are complicated, in general, but for illustration we have one simple result:

$$
\left\langle z^{2}\right\rangle=\frac{1}{2}+\varrho\left(\partial \zeta_{0} / \partial \varrho\right) x \mu_{r \zeta}\left(L_{\eta} \mu_{\eta} / \mu_{\zeta}\right) \nabla \cdot \boldsymbol{u} .
$$

There are also correlations $\langle\xi z\rangle$ and $\langle v z\rangle$ which vanish in equilibrium. In the following section we shall estimate all the terms linear in $\nabla \cdot \boldsymbol{u}$ which result from applying $(24 a, b)$ in $(23 a-e)$.

\section{Order-of-Magnitude Estimates of the Correlation Functions}

Using the kinetic equation for the "hole" model of [18] as extended in the Appendix, Eq. (A.1), to include an inertial term, we shall proceed here to estimate the corrections given in $(23 \mathrm{a}-\mathrm{e})$ to the Einstein approximation to the correlation functions. Since $(23 \mathrm{a}-\mathrm{e})$ are obtained through a truncation approximaion, and since the model proposed in [18] for the structural contribution to sound absorption is crude, these estimates serve to give a rough idea, at best, of the importance of these corrections. Most of them are found to be so small that, even if the 
estimates are off by two or three orders-of-magnitude, they should by negligible in comparison with the Einstein contributions, where the latter are not zero. The results thus resemble those predicted [16] for fluctuations in the heat flow, viz. that the Einstein approximation is excellent so long as the system is not driven by its coupling to the surroundings.

To solve the algebraic equations of Sect. 3.2, we need numerical values of $\mu_{\varrho}, \mu_{\eta}, \mu_{\zeta}$, and $\mu_{r \zeta}$ which are obtained via the procedure outlined in the Appendix and developed in [20]. We shall neglect the contributions to $G$ in Eq. (19) which are not proportional to a thermodynamic force, except for $\tilde{\mu}_{\varrho \eta}$ and $\tilde{\mu}_{\zeta \eta}$ which are proportional to $\mu_{r \zeta}$, since the coefficient of these terms are higher-order entropy derivatives which are assumed small in comparison with the second-order coefficients. $\tilde{\mu}_{\varrho \eta}$ and $\tilde{\mu}_{\zeta \eta}$ are taken equal and so determined that (19) agrees in equilibrium with (10).

Applying the aforementioned approximations, in addition to $(24 \mathrm{a}, \mathrm{b})$ and $(23 \mathrm{a}-\mathrm{e})$, we obtain (25) plus the following:

$$
\begin{aligned}
\left\langle\xi^{2}\right\rangle= & (1 / 4)\left(\tau_{1}^{-1}+\tau_{2}^{-1}\right)+\nabla \cdot \boldsymbol{u} \frac{1}{2} G_{1}\left(\tau_{1} \tau_{2}\right)^{-1 / 2},(26) \\
\langle\xi v\rangle= & (1 / 4)\left(\tau_{1}^{-1}-\tau_{2}^{-1}\right) \\
& +\nabla \cdot \boldsymbol{u}\left[-2 F_{2} \tau_{1}^{-1}+(1 / 4) G_{1}\left(\tau_{1} \tau_{2}\right)^{-1 / 2}\right],(26) \\
\langle\xi z\rangle= & -\nabla \cdot \boldsymbol{u} 2^{-3 / 2} \varrho\left(\partial \zeta_{0} / \partial \varrho\right) \\
& \cdot\left[\left(\mu_{\eta} L_{\eta} / L_{\zeta}\right)\left(D_{3} \tau_{2}^{-1 / 2}+D_{2} \tau_{1}^{-1 / 2}\right)\right. \\
& \left.+\frac{1}{2}\left(E_{3} \tau_{2}^{-1 / 2}+E_{2} \tau_{1}^{-1 / 2}\right)\right], \\
\langle v z\rangle=\nabla & \cdot \boldsymbol{u} 2^{-5 / 2}\left\{\left[-2 \mu_{\eta} \bar{v}_{1} Q_{1}\left(D_{3} \tau_{2}^{-1 / 2}-D_{2} \tau_{1}^{-1 / 2}\right)\right.\right. \\
& \left.\quad-E_{3} \tau_{2}^{-1 / 2}+E_{2} \tau_{1}^{-1 / 2}\right] \varrho\left(\partial \zeta_{0} / \partial \varrho\right) \\
& \left.+G_{3} \tau_{2}^{-1 / 2}-G_{2} \tau_{1}^{-1 / 2}\right\} .
\end{aligned}
$$

The contribution linear in $\nabla \cdot \boldsymbol{u}$ to $\left\langle v^{2}\right\rangle$ vanishes within the approximations used here.

The coefficients $D_{2}, D_{3}, E_{2}, F_{3}, F_{2}$, and $G_{1}$ can be evaluated by applying the abovementioned approximations and methods of Section 3.2. Expressions for the remaining constants are given either in [18] and [21] or in the Appendix, except for $\partial \zeta_{0} / \partial \varrho$. Using Eq. (34) of [18] for $\zeta_{0}$, and $\varepsilon=\delta / L$ with $L=\left(V-V_{0}\right)^{1 / 3}$, where $V_{0}=$ excluded volume, we estimate that

$$
\begin{aligned}
\partial \zeta_{0} / \partial \varrho= & -(2 / \pi) \exp \left[-\chi_{0}^{2}\left(1-2 \beta^{-1}\right)\right] \\
& \cdot\left[\alpha^{*}(\partial L / \partial \varrho)\left(1-2 \beta^{-1}\right)^{1 / 2}\right. \\
& \left.+\gamma_{0} \beta^{-2}\left(1-2 \beta^{-1}\right)^{-1 / 2} \exp \left(\gamma^{*}\right)\left(\gamma^{*} / 3 \varrho\right)\right],
\end{aligned}
$$

$$
\begin{aligned}
\alpha^{*} & =2 \pi\left(m \bar{v}_{1} / h\right)^{1 / 2}, \\
\chi_{0} & =\alpha^{*} \delta \varepsilon, \\
\gamma^{*} & =7 h c /\left[45 F_{1} F_{2} \delta \varkappa T\right] ; \\
F_{1} & =0.875 ; \quad F_{2}=1.285, \\
\beta & =\exp \left(\gamma^{*}\right)+1 .
\end{aligned}
$$

Numerical values are worked out and listed in Table 1 for liquid Ar at $1 \mathrm{~atm}$. and $87 \mathrm{~K}$. This liquid is monatomic, as assumed in the model, and so the scalar $\zeta$ does not have to be coupled to additional scalar variables whic describe relaxation of internal molecular degrees of freedom. It has the drawback that $\zeta_{0}$ and $Q_{1}$ are anomalously large, probably as a result of the small value of $m$ as compared with the heavier molecular masses discussed in [18]. These are, therefore, probably overestimated here, but an overestimate of an order-of-magnitude cannot change the qualitative conclusions about smallness of the corrections to the Einstein approximation to $g$.

For the calculations in Table 1, the system has been chosen to be $1 \mathrm{~cm}$ on a side. For a sound wave with $2 \pi v \sim 10^{3}, \lambda=5.26 \mathrm{~m}$, and so the condition $l \ll \lambda$ is fulfilled. For such a sound wave, if the amplitude $a$ has a typical ultrasonic value $2 \pi a$ /

Table 1. Experimental and calculated constants for liquid $\mathrm{Ar}$ at $87 \mathrm{~K} . \alpha_{p}=$ coefficient of volume expansion; $\beta_{t}=$ isothermal compressibility; $c=$ hypersound velocity; $c_{s}=$ low frequency sound velocity; $N=$ no. atoms in system. Other symbols defined in Sects. 3 and 4. Last six entries in column 2 are the corrections to Einstein approximation.

\begin{tabular}{lllc}
\hline$\tau_{1}$ & 1.000307 & $\tau_{2}$ & 0.999693 \\
$\varrho$ & $1.40 \cdot 10^{3} \mathrm{~kg} \mathrm{~m}^{-3 \mathrm{a}}$ & $\mu_{r} \zeta$ & $-57.9 \mathrm{~s}^{2}$ \\
$m$ & $6.6336 \cdot 10^{-26} \mathrm{~kg}$ & $\partial \zeta_{0} / \partial \varrho$ & $5.07 \cdot 10^{-7} \mathrm{~m}^{3} / \mathrm{kg}$ \\
$\alpha_{p}$ & $4.61 \cdot 10^{-3} \mathrm{~K}^{-1 \mathrm{a}}$ & $D_{2}$ & $-4.86 \cdot 10^{-6} \mathrm{~K} / \mathrm{J}$ \\
$\varrho \beta_{t}$ & $2.84 \cdot 10^{-6} \mathrm{~s}^{2} / \mathrm{m}^{2 \mathrm{~b}}$ & $D_{3}$ & $4.85 \cdot 10^{-6} \mathrm{~K} / \mathrm{J}$ \\
$c_{s}$ & $837 \mathrm{~ms}^{-1 \mathrm{~b}}$ & $E_{2}$ & $2.25 \cdot 10^{-7} \mathrm{~s}$ \\
$c$ & $1300 \mathrm{~ms}^{-1}$ & $E_{3}$ & $2.25 \cdot 10^{-7} \mathrm{~s}$ \\
$N$ & $2.116 \cdot 10^{10 \mathrm{~b}}$ & $F_{2}$ & $1.20 \cdot 10^{-15} \mathrm{~s}$ \\
$\delta$ & $2.87 \cdot 10^{-10} \mathrm{~m}$ & $F_{3}$ & $21.4 \cdot 10^{-16} \mathrm{~s}$ \\
$\varepsilon$ & 0.244 & $G_{1}$ & $-2.88 \cdot 10^{-9} \mathrm{~s}$ \\
$\bar{v}_{1}$ & $1.82 \cdot 10^{12} \mathrm{~Hz}$ & $G_{2}$ & $1.18 \cdot 10^{-3} \mathrm{~s}$ \\
$\gamma^{*}$ & 0.346 & $G_{3}$ & $1.18 \cdot 10^{-3} \mathrm{~s}$ \\
$\alpha^{*}$ & $8.48 \cdot 10^{5} \mathrm{~m}^{-1}$ & $\langle\xi\rangle$ & $-1.43 \cdot 10^{-8}$ \\
$\zeta_{0}$ & 0.564 & $\left\langle v^{2}\right\rangle$ & 0.00 \\
$Q_{1}$ & $2.19 \cdot 10^{-3}$ & $\langle\xi v\rangle$ & $7.20 \cdot 10^{-9}$ \\
$\mu_{\eta} / \chi$ & $5.90 \cdot 10^{2} \mathrm{~s}^{2}$ & $\left\langle z^{2}\right\rangle$ & $2.15 \cdot 10^{-14}$ \\
$\mu_{\zeta} / \varkappa$ & $3.82 \cdot 10^{22}$ & $\langle\xi z\rangle$ & $5.66 \cdot 10^{-10}$ \\
$\mu_{\varrho} / \chi$ & $4.59 \cdot 10^{-22}$ & $\langle v z\rangle$ & $4.16 \cdot 10^{-3}$ \\
\hline
\end{tabular}

a "Properties of materials at low temperature (Phase I)", ed. by V. J. Johnson, Pergamon Press, New York 1961.

b Landolt-Börnstein Tabellen, New Series, Group II, Vol. 5. 
$\lambda \sim 10^{-2}, \nabla \cdot \boldsymbol{u} \sim 10 \mathrm{~s}^{-1}$, which is the value used in preparing the table.

\section{Summary and Discussion}

The present paper, on corrections to the Einstein distribution for coupled fluctuations in the density and the structural functions $A$ and $\dot{A}$, is designed to extend to the case of scalar variables an analogous discussion $[16,17]$ previously applied to fluctuations in the flow of heat and in the temperature. The impetus for these studies was work [22] in the recent literature in which the Einstein function was used $[3,4]$ to calculate correlations in fluctuations from non-equilibrium states. The predictions of the Einstein function were found [4] in the case of heat flow to be exact in the absence of a temperature gradient but to disagree with the Boltzmann equation in the case of steady-state heat transport. What is needed to resolve the question is an equation for the fluctuaton distribution $g$ which we derive here, as in the earlier work $[16,17]$ on heat flow, from the classical Liouville equation. The derivation uses the projection operator techniques of Zwanzig [13, 14] and Grabert [12] to derive an equation for $g$ of Fokker-Planck type.

A solution to the Fokker-Planck equation is explored in Section 3, starting with the Einstein function $\exp \delta^{2} S / x$ in lowest order and adding corrections linear in the thermodynamic forces. These corrections lead to corresponding corrections in the correlation functions listed in $(29 a-e)$, which are linear in the forces, in contrast to the vector case [16] where symmetry requires that they be quadratic. These corrections appear even when the system is decoupled form the surroundings, so that the Einstein distribution does not give exact binary correlation functions in this case, in contrast to the case of heat flow [16], where it failed only in presence of a temperature gradient $\nabla T$.

While the Einstein distribution is inexact, we nevertheless find it to be a good approximation for the model discussed in Section 4. The kinetics of this model have been discussed in [18], and it is extended in the Appendix, Eq. (A.1), to include an inertial term in $\dot{\eta}=\zeta$. To find the Einstein distribution for this model, we must calculate $\mu_{\eta}$, i.e. the coefficient of the $O\left(\eta^{2}\right)$ term in the extended entropy. The procedure for this, following [20], is described in the Appendix. It illustrates the general principle that, if we can calculate the unextended terms in $S$ and possess a kinetic equation derived from the model, then we can calculate the extended terms as well.

The results in Table 1 indicate that, except for $\langle v z\rangle$, the corrections to the Einstein approximation are wholly negligible. In the latter case, the only appreciable contribution arises from the driving term $\nabla \cdot \boldsymbol{u}$ in the Fokker-Planck equation for $g$. This conclusion is shown only for the liquefied noble gases, since in polyatomic liquids there are more important scalar parameters than the structural $\zeta$ postulated in Section 4. These involve mainly the populations of internal vibrational-rotational states which are coupled to the population of "holes", since collisions producing energy exchange between internal and translational degrees of freedom occur mainly in regions with a gas-like structure in which molecules can move more freely. Because polyatomic molecules are much heavier, $\zeta_{0}$ has a much smaller value [18] than the one estimated here. We cannot conclude, therefore, that the Einstein approximation is good in general in the presence of macroscopic temperature and velocity gradients. In fact, when temperature gradients are present, corrections to Einstein can be of the order of the square of the steady-state heat flux [16].

In the process of calculating correlations involving extended variables, we have been able to answer one of the criticisms posed by Keizer [23], who points out that some of the extant formulations of extended theory provide no way of calculating the extended entropy, so that use of the latter in the Einstein function does not permit numerical calculation of correlations involving the dissipative fluxes. A further criticism [23] of the Einstein function is that it is inexact, but we have, via the Fokker-Planck equation for $g$, a method of correcting it or of estimating the error inherent in using it.

The demonstration that the thermodynamic and hydrodynamic pressures are equal starts by multiplying (9) by $v_{1}$ and integrating over the space of $v$, $v_{1}$, and $N$. We confirm that $(5 \mathrm{~d})$ should have no term linear in $\nabla \cdot \boldsymbol{u}$. This answers a question previously discussed $[5,24]$ concerning the case where the kinetic equation for $\zeta$ contains a term in $\nabla \cdot \boldsymbol{u}$ while the thermodynamic pressure depends quadratically on $\zeta-\zeta_{0}$ vs. the case where $\nabla \cdot \boldsymbol{u}$ is absent from the kinetic equation while the pressure is 
linear in $\zeta-\zeta_{0}$. In linear non-equilibrium thermodynamics these case are equivalent [24], but in the non-linear regime they are not. An analogous question has arisen ((22), p. $144 \mathrm{ff}$.) in the extended non-equlibrium chemical kinetics, since a term linear in $\nabla \cdot \boldsymbol{u}$ is allowed by symmetry. By reciprocity, if the equation for $\dot{\eta}$ depends on $\nabla \cdot \boldsymbol{u}$, then $\bar{P}-P$, where $\bar{P}$ is the pressure trace and $P$ the thermodynamic pressure, should depend of $F_{\zeta}$, and then $\bar{P} \neq P$. In the absence of a term linear in $\nabla \cdot \boldsymbol{u}$ in the equation for $\dot{\eta}$, we can set $\bar{P}=P$. We can calculate $P$ from the extended free energy and use it in making predictions about the non-Newtonian character of $\bar{P}$ in non-linear situations.

\section{Appendix}

\section{Evaluation of Parameters in the Einstein Function}

To make numerical estimates of $\mu_{\varrho}, \mu_{\eta}$, and $\mu_{\zeta}$ for the model discussed in Section 4, in order to estimate in turn the magnitudes of the expressions $(23 a-e)$ for the correlation functions, we must take an approximate calculation of the extended entropy $S$. We shall exploit an earlier demonstration [20] to the effect that, if we can find the $\zeta$-dependence of $S$ and also derive a kinetic equation appropriate to the hole model of Sect. 4 for the time-dependence of $\zeta$, then we can determine the $\eta$-dependence of $S$ and thus estimate $\mu_{\eta}$.

A kinetic equation for the volume fraction, $\zeta$, of "holes" or locally expanded regions within the system has been derived in [18] for frequencies $\lesssim 1 \mathrm{MHz}$. This is extended into the hypersonic range by addition of an inertial term to give

$$
\dot{\eta}=-(1 / \tau) \eta-(1 / \tau)\left[k_{2} \zeta-k_{1}(1-\zeta)\right],
$$

where $k_{1}, k_{2}$ are given in [18], Eqs. (28) and (34). We take $Q^{-1}=\bar{v}_{1} Q_{1}$, where $\bar{v}_{1}=45 c / 112 \delta$ is the mean frequency of expansion of a region $\sim \delta=$ $(m / 2 \varrho)^{1 / 3}$, the intermolecular separation [21], and $Q_{1}$ is the probability that an expansion attains the critical size (cf. expression for $\varepsilon$ in [21]) required to permit self-diffusion. Intuitively, $\zeta$ will not change over a time $\ll \tau$. Equation (A.1) can be cast in the form

$$
\dot{\eta}=-(1 / \tau) \eta-\left(k_{1}+k_{2}\right) \tau^{-1}\left(\zeta-\zeta_{0}\right),
$$

where $\zeta_{0}$, given by [18], Eq. (34), is the equilibrium value of $\zeta$.
A crude estimate of $S$ is achieved by observing that, if $\zeta$ is small so that the "holes" do not mutually interfere, then the number of ways of dividing $N$ spherical subvolumes, each approximately of molecular size, into $N$ expanded and $N(1-\zeta)$ unexpanded is $N ! /[(\zeta N) !(N-\zeta N) !]$. In point of fact, the holes do interfere, since two adjacent holes are equivalent to one larger one, and we take this into account by adding a correction, $-a \zeta^{2}$, to $S$ which becomes

$$
\begin{aligned}
S / \varkappa= & \ln N !-\zeta N \ln \zeta N \\
& -(N-\zeta N) \ln (N-\zeta N)-a \zeta^{2} .
\end{aligned}
$$

The constant $a$ is determined to make $\partial S / \partial \zeta=0$ when $\zeta=\zeta_{0}$, i.e.

$$
a=-\left(N / 2 \zeta_{0}\right) \ln \left[\zeta_{0} /\left(1-\zeta_{0}\right)\right] .
$$

This leads to

$\mu_{\zeta} / \varkappa=\left(N / 2 \zeta_{0}\right)\left\{\left(1-\zeta_{0}\right)^{-1}-\ln \left[\zeta_{0}\left(1-\zeta_{0}\right)^{-1}\right]\right\}$.

To find the $\eta$-dependence of $S$ by the method of ref. 20, we compare (5d) with (A.1) to get

$$
\begin{aligned}
L_{\zeta} F_{\zeta} & =-\bar{v}_{1} Q_{1}\left[k_{2} \zeta-k_{1}(1-\zeta)\right] \\
& =-\left(\bar{v}_{1} Q_{1}\right)^{2}\left[\zeta_{0}\left(1-\zeta_{0}\right)\right]^{-1}\left(\zeta-\zeta_{0}\right) .
\end{aligned}
$$

Since $L_{\zeta}=1 / 2 \mu_{\eta}$, we find

$\mu_{\eta} / \varkappa=\frac{1}{2} N\left(\bar{v}_{1} Q_{1}\right)^{-2}\left\{1-\left(1-\zeta_{0}\right) \ln \left[\zeta_{0} /\left(1-\zeta_{0}\right)\right]\right\}$.

Comparing the terms linear in $\eta$ in (5d) and (A.1), we find that

$$
L_{\eta}=\bar{v}_{1} Q_{1} /\left(2 \mu_{\eta}\right) \text {. }
$$

Since (A.6) gives the $\zeta$-dependence of $-\frac{1}{2} \partial^{2} S / \partial \eta^{2}$, we can use it to find

$$
\begin{aligned}
\mu_{r \zeta}= & (6 x)^{-1} \partial^{3} S / \partial \zeta \partial \eta^{2}=(1 / 12)\left(\bar{v}_{1} Q_{1}\right)^{-2} \\
& \cdot N \zeta_{0}\left(1-\zeta_{0}\right)\left[\zeta_{0}^{-2}-\left(1-\zeta_{0}\right)^{-2}\right] .
\end{aligned}
$$

To estimate $\mu_{0}$ from the grand canonical ensemble we note that $f_{1}(N)$ is proportional to

$$
\exp [\beta(N \bar{\mu}-F(V, T, N)] .
$$

Then $\mu_{\varrho}$ stems from the second-order term in the expansion of $F$ in powers of $N-N_{0}$, where $N_{0}$ is the ensemble average. Using the Gibbs-Duhem equation, we find that

$$
\begin{aligned}
\mu_{\varrho} & =(2 T)^{-1} \partial \mu / \partial N=(m / 2 N T) \partial P / \partial \varrho \\
& =(m / 2 \varrho N T) B_{T},
\end{aligned}
$$

where $B_{T}$ is the isothermal bulk modulus. 
[1] I. Müller, Z. Phys. 198, 329 (1967).

[2] G. Lebon, Bull. Acad. Roy. Sci. Belg. 64, 456 (1978).

[3] G. Lebon, D. Jou, and J. Casas-Vasquez, J. Phys. A: Math. Gen. 13, 275 (1980).

[4] D. Jou, J. Casas-Vasquez, and G. Lebon, J. NonEquil. Thermodyn. 4, 349 (1979).

[5] R. E. Nettleton, J. Chem. Phys. 33, 237 (1960).

[6] R. E. Nettleton, Phys. Fluids 3, 216 (1961).

[7] R. E. Nettleton, Phys. Fluids 4, 74 (1963).

[8] R. E. Nettleton, Nuovo Cim. 28,952 (1963).

[9] R. E. Nettleton, Physica 30, 1989 (1964).

[10] D. Jou, J. E. Llebot, and J. Casas-Vasquez, Physica 109 A, 208 (1981)

[11] D. Jou and T. Careta, J. Phys. A: Math. Gen. 15, 3195 (1982).

[12] H. Grabert, Projection Operator Techniques in Nonequilibrium Statistical Mechanics, Springer, Berlin 1982.
[13] R. Zwanzig, J. Chem. Phys. 33, 1338 (1960).

[14] R. Zwanzig, Phys. Rev. 124, 983 (1961).

[15] R. E. Nettleton, J. Chem. Phys. 40, 112 (1964).

[16] R. E. Nettleton, J. Phys. A: Math. Gen. 17, 2149 (1984).

[17] R. E. Nettleton, J. Chem. Phys. 81, 2458 (1984).

[18] R. E. Nettleton, J. Acoust. Soc. Amer. 31, 557 (1959).

[19] M. Mooney, Trans. Soc. Rheol. (USA) 1, 63 (1957).

[20] R. E. Nettleton, Phys. Letters 24 A, 231 (1967).

[21] R. E. Nettleton, Phys. Fluids 2, 256 (1959).

[22] Proc. Int. Seminar on "Recent Developments in Nonequilibrium Thermodynamics", Springer, Berlin 1984, see p. $105 \mathrm{ff}$.

[23] J. Keizer, J. Stat. Phys. 31, 485 (1983).

[24] R. E. Nettleton, Phys. Fluids 4, 1488 (1961). 